\title{
¿EXISTEN COMPONENTES PRONOSTICABLES EN LAS SERIES DE LOS RENDIMIENTOS DE LAS ACCIONES?
}

\author{
José Carlos Ramírez*
}

División de Economía, Centro de Investigación y Docencia Económicas, A. C. Rogelio Sandoval-Saavedra

División de Economía, Centro de Investigación y Docencia Económicas, A. C.

(Recibido 21 de mayo 2001, aceptado 20 de febrero 2002)

\begin{abstract}
Resumen
En este trabajo, la prueba desarrollada por McQueen y Thorley (1991) para caminatas aleatorias se aplica, con algunas modificaciones, a títulos de capital negociados en la Bolsa Mexicana de Valores. Para tal efecto, se examinan los rendimientos diarios de un portafolio compuesto por acciones de alta bursatilidad mediante una cadena de Markov de segundo orden, con lo cual se evita el supuesto de normalidad. Se presentan y discuten los resultados del análisis empírico y las pruebas sobre las probabilidades de transición. Asimismo, se investiga si la cadena de Markov es ergódica y por lo tanto estacionaria (en covarianza). Finalmente, se calculan algunos pronósticos basados en las estimaciones obtenidas.
\end{abstract}

\begin{abstract}
In this paper, the procedure developed by McQueen and Thorley (1991) to test for a random walk is applied, with some modifications, to the securities traded in the Mexican Stock Exchange. In order to, the daily returns of a portfolio made up of highly traded securities are examined by means of a second-order Markov chain avoiding the assumption of normality. The results of the estimation"and the tests on the transition probabilities are presented and discussed. Also, it is investigated if the Markov chain is ergodic and therefore (covariance) stationary. Finally, forecasts based on the obtained estimations are provided.
\end{abstract}

Clasificación JEL: C1, G1.

Palabras Claves: Econometric and Statistical Methods, Financial Markets.

* División de Economía, Centro de Investigación y Docencia Económicas, A. C., Carretera México-Toluca 3655 (km. 16.5), Lomas de Santa Fe, 01210 México, D. F., Teléfono (52) 57279800, E-mail: josecarlos.ramirez@cide.edu.

Los autores desean agradecer a dos dictaminadores anónimos sus valiosas observaciones. Asimismo, los autores agradecen al Dr. Francisco Venegas-Martínez sus acertados comentarios en beneficio del presente trabajo. 


\section{Introducción}

Bajo el supuesto de "eficiencia absoluta" de un mercado, las series de los rendimientos de las acciones siguen un comportamiento descrito por una caminata aleatoria sin tendencia, lo cual equivale a decir que no hay componentes pronosticables en las series. La forma de verificar si esta propiedad se cumple consiste en aplicar pruebas estadísticas que revelen el grado de autocorrelación entre los rendimientos, como las presentadas por Fama y French (1988) y Lo y Mackinlay (1988) en dos de los trabajos clásicos sobre el tema. Las pruebas estadísticas en estos trabajos establecen que, en caso de que los índices rezagados de autocorrelación no difieran significativamente de la hipótesis nula de caminata aleatoria, entonces no es posible desarrollar pronósticos con base en los datos históricos.

El grueso de las investigaciones hechas para los países desarrollados reporta la existencia de autocorrelación negativa en las series de los rendimientos anuales y positiva en la de los rendimientos semanales, con lo cual se concluye, en términos generales, que existen en tales series componentes transitorios $y$, por lo tanto, predecibles (véase por ejemplo el trabajo de Campbell et al., 1997). El rechazo sistemático a la hipótesis de la caminata aleatoria en estas investigaciones no implica la violación del supuesto de "eficiencia relativa" de un mercado, puesto que es posible que algunos inversionistas desarrollen sus pronósticos sobre la base de una tecnología de información superior al promedio, lo que les permitiría realizar arbitraje. ${ }^{1}$

Algunos autores como McQueen y Thorley (1991) hacen una crítica a las pruebas estadísticas que utilizan los trabajos anteriores porque no toman en cuenta: 1) las burbujas especulativas, 2) el efecto de las noticias sobre el precio de las acciones y 3) la variación temporal de los rendimientos, presentes en cualquier mercado financiero. Estos elementos, a decir de los autores, producen patrones no lineales en el comportamiento de los rendimientos que no son captados por las pruebas tradicionales que suponen linealidad. Para salvar este obstáculo, sugieren la construcción de pruebas alternativas en las que la hipótesis de la caminata aleatoria esté contenida en la estructura de probabilidades de transición de una cadena de Markov. De tal suerte que, si la hipótesis no es rechazada, entonces las probabilidades no tendrían estructura y lòs rendimientos presentes de las acciones serían igualmente probablès sin importar la historia.

El objetivo de este trabajo es aplicar, con algunas modificaciones, la prueba desarrollada por McQueen y Thorley (1991) a títulos de capital negociados en la Bolsa Mexicana de Valores. Para tal efecto, se utilizan los rendimientos diarios de un portafolio compuesto por acciones de alta bursatilidad a fin de evitar el problema de correlación espuria causada por el comercio infrecuente

1 Debido a la confusión que puede introducir la idea de pronósticos en mercados eficientes, Lo (1997) recomienda utilizar en lugar del supuesto de eficiencia absoluta el de eficiencia relativa, ya que este úitimo acepta la existencia de arbitraje cuando se presentan "ineficiencias" de mercado y no cuando son producto de ventajas competitivas permanentes como puede ser el tener una capacidad superior de innovación financiera. 
de títulos. La elaboración de este ejercicio se justifica no sólo por los innumerables problemas técnicos asociados con los modelos lineales heteroscedásticos tan comúnmente usados en finanzas, que además arrojan resultados poco robustos para el caso mexicano (veáse, al respecto a Ramírez, 2001), sino porque los métodos estocásticos no lineales ofrecen una solución alternativa más convincente a la falta de normalidad que presentan los rendimientos diarios de las acciones (veáse Venegas-Martínez, 2001 y Lorenzo-Váldes, 2002). En este contexto, la conclusión principal del presente trabajo, que consiste en rechazar la hipótesis de la caminata aleatoria para las series de datos de rendimientos empleadas, sugiere la existencia de un patrón de pronósticos de las acciones mexicanas, el cual no necesariamente se ajusta a la práctica habitual de corrección del sesgo y exceso de curtósis de las series. Además, con tal resultado se evitan los problemas de especificación, ajuste y parsimonia de los modelos heteroscedásticos.

Finalmente, el trabajo está organizado de la siguiente manera. En la segunda sección se presenta un modelo, desarrollado a partir de los trabajos de Neftci (1984), Falk (1986) y McQueen y Thorley (1991), en el cual se asocia a los rendimientos de las acciones una cadena de Markov de segundo orden. En la tercera sección, se presentan los resultados de la estimación y las pruebas sobre las probabilidades de transición. En la cuarta sección se calculan las probabilidades de equilibrio de la cadena Markoviana y se investiga si esta es ergódica y, por lo tanto, estacionaria. En la quinta sección se generan las series de pronósticos y, por ultimo, en la sexta sección, se presentan las principales conclusiones y posibles extensiones del trabajo.

\section{El Modelo}

A continuación se presenta un modelo que tiene su origen en los trabajos de ciclos económicos desarrollados por Neftci (1984) y Falk (1986). En estos trabajos, los autores evalúan la asimetría de los ciclos económicos bajo la hipótesis de que si las series son simétricas a lo largo del ciclo, las probabilidades de transición entre los estados de la cadena de Markov asociada deben ser iguales. McQueen y Thorley (1991) extienden este razonamiento para probar la existencia de una caminata aleatoria en' las series de rendimientos de las acciones cotizadas y negociadas en la Bolsa de Valores de Nueva York. De acuerdo con esta idea, McQueen y Thorley argumentan que si los rendimientos siguen una caminata aleatoria, entonces las probabilidades de transición de la cadena tienen que ser también iguales. En caso contrario, los rendimientos presentes pueden ser pronosticados con base en la probabilidad de los rendimientos bajos o altos registrados en periodos anteriores más no en los rendimientos pasados en sí. Lo anterior resulta ser conveniente, dado que esto nos ayuda a evitar los problemas asociados con la falta de normalidad multivariada común en las series de rendimientos de acciones. ${ }^{2}$ Recordemos, que la razón por la cual el supuesto de normalidad es insostenible en este tipo de datos, es porque tales series generalmente presentán sesgos y excesos de curtósis. Sin embargo, con el

2 Véase a Ramírez (2001) y Venegas-Martinez (2001) para el caso de México. 
uso de una cadena de Markov, las series de los rendimientos no requieren seguir una distribución normal, siempre y cuando la cadena asociada sea estacionaria.

La estructura de un modelo de este tipo, requiere primero ordenar la serie de rendimientos de acuerdo con una secuencia $I_{t}$ definida de la siguiente forma:

$$
I_{t}=\left\{\begin{array}{lll}
1, & \text { si } & r_{t}>R \\
0, & \text { si } & r_{t}<R
\end{array}\right.
$$

donde $r_{t}=\ln \left(P_{t} / P_{t-m}\right)$ es el rendimiento de cierto activo en el tiempo $t$ cuyo precio se define como $P_{t}$. Por otra parte, supondremos de aquí en adelante, $m=1$ para rendimientos diarios, $m=5$ para semanales y así sucesivamente. $R$ representa el rendimiento promedio de dichas acciones; en otras palabras, es una medida de la esperanza matemática. Los rendimientos aparecen así divididos en rendimientos altos y bajos con respecto a $R$.

Ahora bien, considerando a $S_{T}=\left\{i_{1}, i_{2}, \ldots, i_{T}\right\}$ como una realización de $I_{t}$, podemos definir la función de verosimilitud de la cadena de Markov de segundo orden como: ${ }^{3}$

$$
\begin{aligned}
L\left(S_{T}\right)= & \operatorname{Pr}\left\{I_{2}=i_{2}, I_{1}=i_{1}\right\} \times \\
& \prod_{s=0}^{T-3} \operatorname{Pr}\left\{I_{T-s}=i_{T-s} \mid I_{T-s-2}=i_{T-s-2}, I_{T-s-1}=i_{T-s-1}\right\}
\end{aligned}
$$

la cual, incluyendo las probabilidades del estado inicial, $\operatorname{Pr}\left\{I_{2}=i_{2}, I_{1}=i_{1}\right\}=$ $\pi_{0}$, y las de transición, $\operatorname{Pr}\left\{I_{k}=0 \mid I_{k-2}=i, I_{k-1}=j\right\}=\lambda_{i j}$, se puede reescribir como:

$$
L\left(S_{T}, \Lambda, \pi_{0}\right)=\pi_{0} \prod_{i j=00}^{1} \lambda_{i j}^{N_{i j}}\left(1-\lambda_{i j}\right)^{M_{i j}}, \quad i, j=0,1
$$

donde

$$
\Lambda=\left[\lambda_{00}, \lambda_{01}, \lambda_{10}, \lambda_{11}\right],
$$

$N_{i j}$ representa el número de secuencias de ceros y unos, tal que se cumple $\left\{I_{t}=0 \mid I_{t-2}=i, I_{t-1}=j\right\}, i, j=0,1$ y $t=3,4, \ldots, T$, y $M_{i j}$ el número de secuencias con el patrón $\left\{I_{t}=1 \mid I_{t-2}=i, I_{t-1}=j\right\}, i, j=0,1$ y $t=3,4, \ldots, T$. Finalmente, la forma logarítmica (o función de log-verosimilitud) de la anterior ecuación es:

$$
\ln \left[L\left(S_{T}, \Lambda, \pi_{0}\right)\right]=\ln \left(\pi_{0}\right)+\sum_{i j=0}^{11}\left\{N_{i j} \ln \left(\lambda_{i j}\right)+M_{i j} \ln \left(1-\lambda_{i j}\right)\right\} .
$$

3 En la siguiente sección se jutificará la elección del orden de la cadena de Markov apoyada en el criterio de información de Schwarz y en evidencia experimental. 
Como se puede observar, la función de log-verosimilitud depende de las probabilidades de transición y de la probabilidad del estado inicial. ${ }^{4}$ Esto puede representar un problema ya que tomar en cuenta el estado inicial implica utilizar métodos numéricos engorrosos para obtener los estimadores máximo verosímiles. Por esta razón, y con el fin de obviar el cálculo de la probabilidad del estado inicial en la estimación, supondremos que el número de observaciones es grande. De esta manera, al resolver las condiciones de primer orden del problema de maximización de $\ln (L)$ e ignorando $\pi_{0}$, se obtienen los siguientes estimadores:

$$
\lambda_{i j}=\frac{N_{i j}}{N_{i j}+M_{i j}}, \quad i, j=0,1 .
$$

Las matrices de ocurrencias y de probabilidades de transición de la cadena de Markov de segundo orden se resumen en el cuadro $1 .{ }^{5}$

Cuadro 1. Ocurrencias y probabilidades de transición.

\begin{tabular}{|c|c|c|c|c|c|c|c|}
\hline \multicolumn{2}{|c|}{$\begin{array}{c}\text { Estados } \\
\text { Anteriores }\end{array}$} & & \multicolumn{2}{c|}{$\begin{array}{c}\text { Ocurrencias } \\
\text { Estado actual }\end{array}$} & & \multicolumn{2}{c|}{$\begin{array}{c}\text { Probabilidades } \\
\text { Estado actual }\end{array}$} \\
\hline & & & 0 & 1 & & 0 & 1 \\
\hline 0 & 0 & & $N_{00}$ & $M_{00}$ & & $\lambda_{00}$ & $1-\lambda_{00}$ \\
\hline $0^{*}$ & 1 & & $N_{01}$ & $M_{01}$ & & $\lambda_{01}$ & $1-\lambda_{01}$ \\
\hline 1 & 0 & & $N_{10}$ & $M_{10}$ & & $\lambda_{10}$ & $1-\lambda_{10}$ \\
\hline 1 & 1 & & $N_{11}$ & $M_{11}$ & & $\lambda_{11}$ & $1-\lambda_{11}$ \\
\hline
\end{tabular}

La prueba de hipótesis de la caminata aleatoria se reduce en este contexto a hacer un contraste de igualdad entre todas las probabilidades de transición o a comparar las probabilidades $\lambda_{00}$ y $\lambda_{11}$, como una opción sugerida por la posible presencia de burbujas racionales. Los estadísticos comúnmente utilizados para tal efecto son la de razón de verosimilitud y la prueba de Wald, que al igual que el multiplicador de Lagrange se distribuye asintóticamente como una $\chi^{2}$ con un número de grados de libertad igual a las $k$ restricciones existentes. El estadístico de razón de verosimilitud se define como sigue:

$$
R V=2[\ln (L(\hat{\Lambda}))-\ln (L(\tilde{\Lambda}))] \approx \chi_{k}^{2}
$$

donde $\ln (L(\hat{\Lambda}))$ es la función de verosimilitud evaluada en los estimadores irrestrictos y $\ln (L(\tilde{\Lambda}))$ es evaluada en la restricción. La región de confianza de la hipótesis $\lambda_{00}=\tilde{\lambda}_{00}, \quad \lambda_{11}=\tilde{\lambda}_{11}$ se puede graficar como el contorno de una

4 Nefcti (1984) demuestra que $\pi_{0}$ se puede expresar como una función de los $\lambda_{i j}$ 's, y la estimación de las probabilidades de transición requiere de métodos iterativos.

5 Más adelante se demostrará cómo esta cadena de Markov de segundo orden se puede representar con una matriz de transición de una cadena de primer orden, donde los diferentes estados vienen dados por las realizaciones de una cadena de segundo orden. 
$\chi^{2}$ con 2 grados de libertad y con un nivel de significancia definido según la siguiente relación:

$$
2[\ln (L(\hat{\Lambda}))-\ln (L(\tilde{\Lambda}))]=\chi_{2}^{2}(1-\alpha) .
$$

Asimismo, la prueba de Wald para la hipótesis $H_{0}: \lambda_{00}=\lambda_{11}$ se define como sigue:

$$
P W=\frac{\left(\hat{\lambda}_{00}-\hat{\lambda}_{11}\right)^{2}}{\sigma^{2}\left(\hat{\lambda}_{00}\right)-\sigma^{2}\left(\hat{\lambda}_{11}\right)} \approx \chi_{1}^{2}
$$

donde

$$
\sigma^{2}\left(\lambda_{i j}\right)=\frac{\lambda_{i j}\left(1-\lambda_{i j}\right)}{N_{i j}+M_{i j}} .
$$

\section{Estimación y Pruebas}

Para llevar a cabo la estimación del modelo se utilizan los rendimientos de 21 títulos de capital de alta bursatilidad cotizados y negociados en la Bolsa Mexicana de Valores. Los rendimientos diarios (días hábiles) se calculan como $r_{t}=\ln \left(P_{t} / P_{t-1}\right)$. El periodo muestral comprende del 03/1/1994 al 24/2/2000. La serie analizada es el rendimiento nominal de un portafolio con ponderaciones iguales para los 21 activos elegidos. El cuadro 2 detalla los títulos, su volumen diario promedio y su tasa de comercio, calculada como el porcentaje de días en la muestra que registraron actividad.

Los rendimientos del portafolio a lo largo del periodo muestral se presentan en la Gráfica 1. Como se aprecia, la serie fluctúa consistentemente alrededor del cero y su varianza no es constante a lo largo del tiempo. Las Gráficas 2 y 3 muestran las propiedades de la distríbución de los rendimientos de la cartera y, finalmente, el Cuadro 3 reporta sus estadísticos descriptivos.

En ese orden, la segunda gráfica presenta el histograma de la serie, que como era de esperarse se encuentra centrado en el cero con un ligero sesgo positivo y una curtósis con un valor de 10.42170 (lo que es indicativo de su exceso con respecto a una normal estándar). La Gráfica 3 nos muestra la estimación del kernel de Epanechnikov de la densidad de la serie, usando un ancho de banda de Silverman con valor $h=0,0066$. Aquí, de nuevo se puede observar el exceso de curtosis de los rendimientos. Este hecho se confirma con la prueba de normalidad de Jarque-Bera, que rechaza enfáticamente la normalidad de los datos. Vale la pena destacar que en Ramírez (2001), al analizar la distribución de las series individuales de rendimientos de los activos de esta cartera, se concluye que no tienen una distribución normal multivariada. Los resultados sugieren, entonces, que una prueba que supone de inicio normalidad estaría sesgada, por lo que la aplicación del contraste de McQueen y Thorley parece adecuado. 
Cuadro 2. Activos del portafolio.

\begin{tabular}{|c|c|c|}
\hline Activo & $\begin{array}{c}\text { Volumen } \\
\text { Promedio }\end{array}$ & $\begin{array}{c}\text { Actividad } \\
(\%)\end{array}$ \\
\hline ALFA.A & 1047149 & 99,55 \\
\hline APASCO & 435828 & 99,87 \\
\hline BANACCI.O & 2162045 & 99,55 \\
\hline BIMBO.A & 340843 & 94.55 \\
\hline CEMEX.CPO & 1667267 & 99.87 \\
\hline CIFRA.V & 2754756 & 99,87 \\
\hline COMERCI.UBC & 1315604 & 99,42 \\
\hline DESC.B & 557136 & 98,44 \\
\hline ELEKTRA.CPO & 832660 & 95,14 \\
\hline FEMSA.UBD & 1646087 & 99,94 \\
\hline GCARSO.A1 & 1524607 & 100,00 \\
\hline GFNORTE.O & 446300 & 86.38 \\
\hline GMEXICO.B & 690932 & 96.99 \\
\hline ICA & 693675 & 99.81 \\
\hline SAVIA.A & 671616 & 99.61 \\
\hline SORIANA.B & 485192 & 94.75 \\
\hline TAMSA & 112822 & 87.61 \\
\hline TELECOM.A1 & 514651 & 58.30 \\
\hline TELMEX.L & 15567421 & 99,94 \\
\hline TELEVISA.CPO & 225522 & 99.68 \\
\hline VITRO.A & 721581 & 99,74 \\
\hline & & \\
\hline & & \\
\hline
\end{tabular}

Gráfica 1. Rendimientos del portafolio.

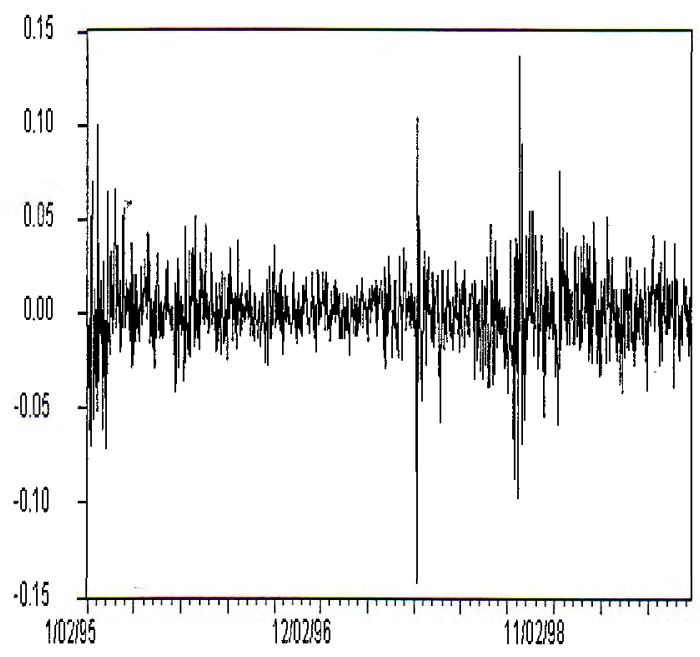


Gráfica 2 . Histograma de los rendimientos de lá cartera.

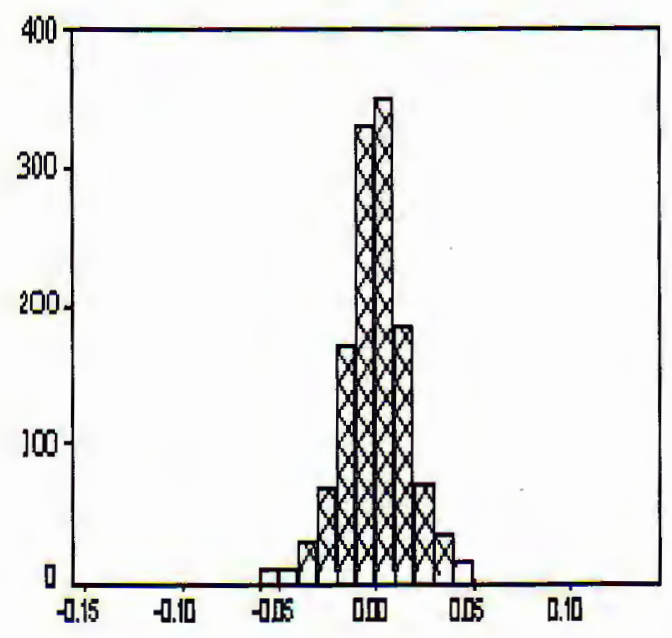

Gráfica 3. Distribución estimada de los rendimientos de la cartera.

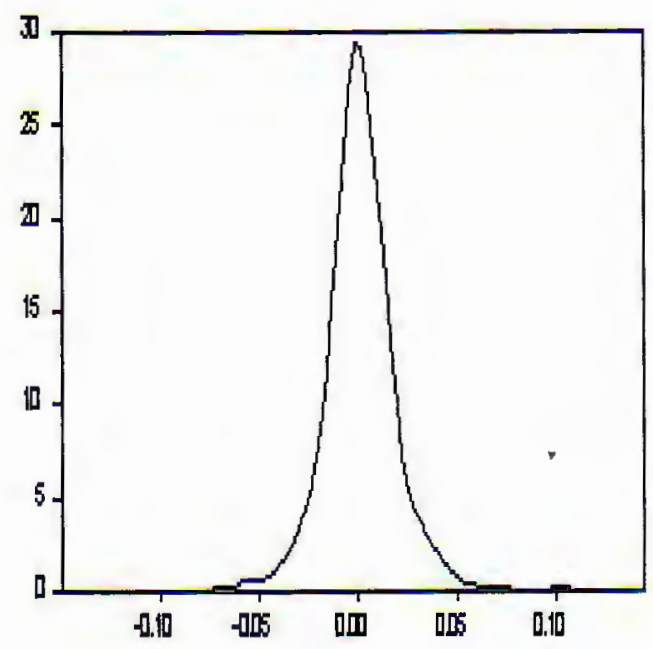


Cuadro 3. Estadísticos descriptivos de los rendimientos de la cartera.

\begin{tabular}{|c|c|}
\hline Estadístico & Valor \\
\hline Media & 0.000753 \\
\hline Mediana & 0.000776 \\
\hline Desv. Estándar & 0.018948 \\
\hline Sesgo & 0.059838 \\
\hline Curtosis & 10.42170 \\
\hline
\end{tabular}

La medida de expectativas que se utilizó en el presente trabajo para dividir a los datos en altos y bajos, consiste en un promedio móvil de los rendimientos diarios del año anterior. Al utilizar esta media móvil, se construyó la serie $\left\{I_{t}\right\}$ de unos y ceros, y se procedió a clasificar los rendimientos de acuerdo con los valores de las realizaciones pasadas. Por otra parte, con el propósito de encontrar el grado óptimo de la cadena de Markov se utilizó el criterio de información de Schwarz $(C S)$, definido de la siguiente manera:

$$
C S=\frac{-2 \ln (L)}{T}+\frac{k \ln (T)}{T}
$$

donde

$$
k=2^{p}+2^{p-1}\left(2^{p}-1\right),
$$

aquí $p$ es el orden de la cadena de Markov, $T$ es el número de observaciones y $\ln (L)$ es la función de log-verosimilitud evaluada en los parámetros que la maximizan. El número de parámetros a estimar, $k$, es la suma de los estimados de las probabilidades de transición (primer término de la suma) ${ }^{6}$ y sus varianzas y covarianzas (segundo término). El criterio de selección para esta formulación del $C S$ consiste en tomar el modelo con el menor valor absoluto del estadístico. El Cuadro 4 nos muestra los valores para la cadena de Markov de primero, segundo y tercer orden.

Cuadro 4. CS para distintos órdenes de la cadena de Markov.

\begin{tabular}{|c|c|c|c|c|}
\hline$p$ & $\ln (L)$ & $T$ & $k$ & $C S$ \\
\hline 1 & -721.10 & 1291 & 3 & 1,1338 \\
\hline 2 & -699.22 & 1290 & 10 & 1,1396 \\
\hline 3 & -684.59 & 1289 & 36 & 1,2622 \\
\hline
\end{tabular}

Al observar los resultados reportados en el cuadro anterior, y siguiendo el criterio de minimizar el valor de $C S$, podríamos decir en principio que la cadena de primer orden es la óptima. Sin embargo, se puede constatar también que la función de log-verosimilitud de la cadena de primer orden es menor que la de la cadena de segundo orden, además de que la diferencia entre los $C S$ de ambas es mínima. Lo que significa que la pérdida de parsimonia debida al mayor

6 En este caso, no se contabilizan las medias ya que la media muestral de una distribución Bernoulli con probabilidad de éxito $p$ es el estimador mismo de $p$. 
número de parámetros a estimar en el proceso de segundo orden no hace menos verosímiles a sus estimadores. Por último, si uno observa los resultados para la cadena de tercer orden, la pérdida de parsimonia es bastante más grande que en las primeras dos (el $C S$ es igual a 1.2622), a pesar de que la función de log-verosimilitud evaluada en sus parámetros es la mayor de las tres. Todo lo anterior, aunado a la evidencia empírica citada en el trabajo de McQueen y Thorley $(1991)^{7}$, nos lleva a considerar que el orden adecuado para la cadena de Markov en este experimento en particular es el segundo.

Cuadro 5. Ocurrencias y probabilidades de transición estimadas.

\begin{tabular}{|c|c|c|c|c|c|c|c|}
\hline $\begin{array}{c}\text { Estados } \\
\text { Anteriores }\end{array}$ & & \multicolumn{2}{c|}{$\begin{array}{c}\text { Ocurrencias } \\
\text { Estado actual }\end{array}$} & & \multicolumn{2}{c|}{$\begin{array}{c}\text { Probabilidades } \\
\text { Estado actual }\end{array}$} \\
\hline & & & 0 & 1 & & 0 & 1 \\
\hline 0 & 0 & & 160 & 107 & & 0,5993 & 0,4007 \\
\hline 0 & 1 & & 67 & 105 & & 0,3895 & 0,6105 \\
\hline 1 & 0 & & 106 & 104 & & 0,6199 & 0,3801 \\
\hline 1 & 1 & & 104 & 576 & & 0,1529 & 0,8471 \\
\hline
\end{tabular}

\begin{tabular}{|c|c|}
\hline \multicolumn{2}{|c|}{$\begin{array}{l}\text { Desviaciones Estándar de las } \\
\text { Probabilidades de Transición }\end{array}$} \\
\hline$\sigma\left(\lambda_{00}\right)$ & 0,02999 \\
\hline$\sigma\left(\lambda_{01}\right)$ & 0,03718 \\
\hline$\sigma\left(\lambda_{10}\right)$ & 0,03712 \\
\hline$\sigma\left(\lambda_{11}\right)$ & 0,01380 \\
\hline
\end{tabular}

El cuadro 5 reporta la estimación de las probabilidades de transición y sus desviaciones estándar. La estimación de estas probabilidades revela una fuerte correlación positiva en los rendimientos de la cartera, es decir: la probabilidad de obtener un rendimiento bajo luego de haber tenido una secuencia de dos rendimientos bajos, es mayor a ła de obtener un rendimiento bajo luego de dos rendimientos altos. Este resultado es similar a los obtenidos por McQueen y Thorley (1991) y Lo y MacKinlay (1988) en sus respectivos análisis con series semanales de rendimientos. Sin embargo, habrá que decir que la alta frecuencia de los datos parece inducir este comportamiento, puesto que al analizar rendimientos anuales McQueen y Thorley encuentran una fuerte correlación negativa, lo cual es un resultado bastante común en la literatura sobre finanzas conocido como "función característica de autocorrelación" de los rendimientos.

Por otra parte, para analizar la significancia de esta correlación y probar así la hipótesis del camino aleatorio, desarrollamos ahora las pruebas de razón de verosimilitud $(R V)$ y de la Wald. El cuadro 6 presenta los resultados.

7 Los autores citan los resultados obtenidos por Kroll, Levy y Rapoport (1988), quienes concluyen que los inversionistas se comportan como si su memoria fuera de dos periodos. 
Cuadro 6. Pruebas de hipótesis sobre las probabilidades de transición del proceso de Markov de segundo orden.

\begin{tabular}{|c|c|c|c|c|c|c|}
\hline$H_{0}$ & $R V$ & $p$-value & Wald & $p$-value & $\chi_{1}^{2}(5 \%)$ & $\chi_{1}^{2}(1 \%)$ \\
\hline$\lambda_{00}=\lambda_{11}$ & 179.53 & 0.00 & 182.75 & 0.00 & 3.84 & 6.63 \\
\hline
\end{tabular}

\begin{tabular}{|c|c|c|c|c|}
\hline$H_{0}$ & $R V$ & $p$-value & $\chi_{1}^{2}(5 \%)$ & $\chi_{1}^{2}(1 \%)$ \\
\hline$\lambda_{00}=\lambda_{01}=\lambda_{10}=\lambda_{11}$ & 253.31 & 0.00 & 7.82 & 11.34 \\
\hline
\end{tabular}

De acuerdo con los resultados del cuadro 6, las dos hipótesis nulas son enfáticamente rechazadas. La primera es rechazada tanto por la prueba de razón de verosimilitud como por la prueba Wald. Los valores de los estadísticos de prueba son muy similares, por lo que podemos confiar en su comportamiento asintótico. En seguida, la segunda hipótesis es tambien rechạzada con fuerza, de tal forma que podemos concluir que los rendimientos no se comportan como una caminata aleatoria ya que la correlación positiva que presentan es significativa. Estos resultados son confirmados por dos pruebas adicionales. La primera de ellas aparece en la gráfica 4 donde se presenta la región de confianza para la hipótesis nula $H_{0}: \lambda_{00}=\tilde{\lambda}_{00}, \lambda_{11}=\tilde{\lambda}_{11}$.

Gráfica 4. Región de confianza para $H_{0}: \lambda_{00}=\tilde{\lambda}_{00}, \lambda_{11}=\tilde{\lambda}_{11}$.

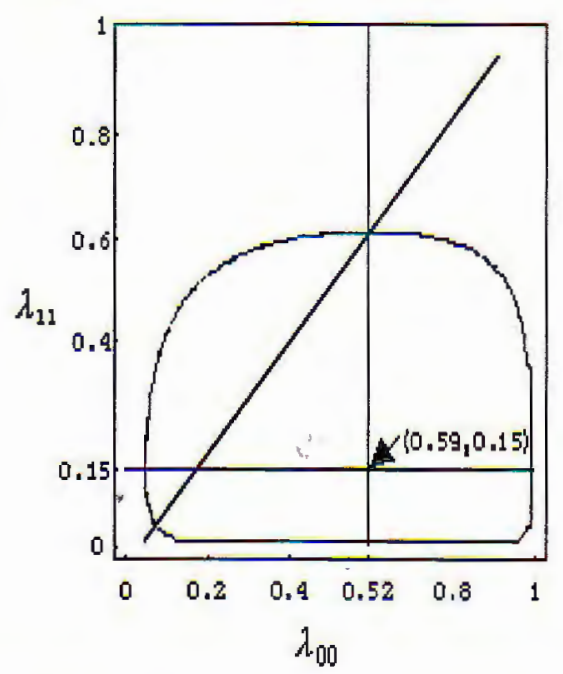

Esta prueba revela gráficamente la existencia de correlación positiva entre los rendimientos de la cartera. La recta de $45^{\circ}$ indica la restricción o aquellas combinaciones de $\lambda_{00}$ y $\lambda_{11}$ que son idénticas. Entonces, valores por encima de la recta representan puntos donde $\lambda_{00}<\lambda_{11}$, reflejando con ello una correlación negativa, pues es mayor la probabilidad de obtener rendimientos altos luego de una secuencia de dos bajos. A su vez, los puntos por debajo de la recta revelan una correlación positiva. 
Las regiónes representan el contorno de $2[\ln (L(\hat{\lambda}))-\ln (L(\tilde{\lambda}))]=\chi_{2}^{2}(1-\alpha)$, donde $\alpha=1 \%$ en el área mayor y $\alpha=10 \%$ en la que se encuentra dentro de ella. En este caso, el énfasis con el que se rechaza la hipótesis nula es mucho menor, debido a que una parte de los elipsoides cruza la recta de $45^{\circ}$. Sin embargo, como podemos observar, la mayor parte de su area se encuentra dentro de la región inferior de la gráfica.

Se hizo una prueba adicional, esta vez apoyada en los criterios de las medias condicionales de los rendimientos del portafolio. La prueba que se realizó es aquella conocida como la de diferencia de medias, la cual se distribuye como una $F$ con grados de libertad en el numerador igual al número de medias comparadas menos uno y en el denominador igual al total de observaciones menos el número de las medias que se compararon. La prueba consistió en condicionar a los rendimientos de los dos periodos pasados usando la media móvil de un año para dividirlos en altos y bajos. En este caso, $R_{10}$ será la media de los activos que tuvieron un rendimiento alto dos periodos atrás y uno bajo en el anterior, y así sucesivamente. Los resultados son reportados en el Cuadro 7.

Cuadro 7. Medias condicionales y pruebas de igualdad entre ellas.

\begin{tabular}{|c|c|c|c|c|}
\hline & Ocurrencias & Media & $\begin{array}{c}\text { Desviación } \\
\text { Estándar }\end{array}$ & $\begin{array}{c}\text { Desviación } \\
\text { Estándar de la } \\
\text { Media Condicional }\end{array}$ \\
\hline$R_{00}$ & 380 & -0.002124 & 0.017983 & 0.001101 \\
\hline$R_{01}$ & 275 & 0.003924 & 0.012710 & 0.000969 \\
\hline$R_{10}$ & 275 & -0.001982 & 0.012607 & 0.000964 \\
\hline$R_{11}$ & 360 & 0.001842 & 0.021545 & 0.000826 \\
\hline
\end{tabular}

\begin{tabular}{|c|c|c|c|}
\hline$H_{0}$ & Grados de Libertad & Estadístico $F$ & $p$-value \\
\hline$R_{00}=R_{11}$ & $(1,738)$ & 7.10 & 0.007 \\
\hline$R_{00}=R_{11}=R_{10}=R_{01}$ & $(3,1286)$ & 5.66 & 0.000 \\
\hline
\end{tabular}

De nueva cuenta, se puede observar evidencia de correlación positiva. Esto ocurre, debido a que la media condicionada en dos rendimientos bajos anteriores es negativa y la condicionada en dos rendimientos altos es positiva, con lo cual se obtienen rendimientos bajos si los dos anteriores han sido bajos y altos si los dos anteriores han sido altos. Las hipótesis nulas se rechazan en ambas pruebas, de tal manera que se confirman los resultados obtenidos al analizar las probabilidades de transición.

Por último, y dado que la elección de la medida de expectativas es un componente crucial en esta prueba, se realizó un análisis de sensibilidad entre distintas medidas a fin de mostrar la robustez de los resultados. Para este propósito, se tomaron medias móviles de 6, 15 y 18 meses, además de los resultados reportados a 12 meses, y se construyeron las correspondientes series $I_{t}$, con las cuales se estimaron las probabilidades de transición y los estadísticos de prueba. Las Gráficas 5 y 6 muestran los estimadores de $\lambda_{00}$ y $\lambda_{11}$ 
para las distintas longitudes del promedio móvil, además de presentar bandas de \pm 2 desviaciones estándar. En ambas gráficas, podemos apreciar que los estimadores no tienen cambios abruptos, así como también que las desviaciones no se alejan demasiado entre sí.

Gráfica 5. Análisis de sensibilidad de $\lambda_{00}$.

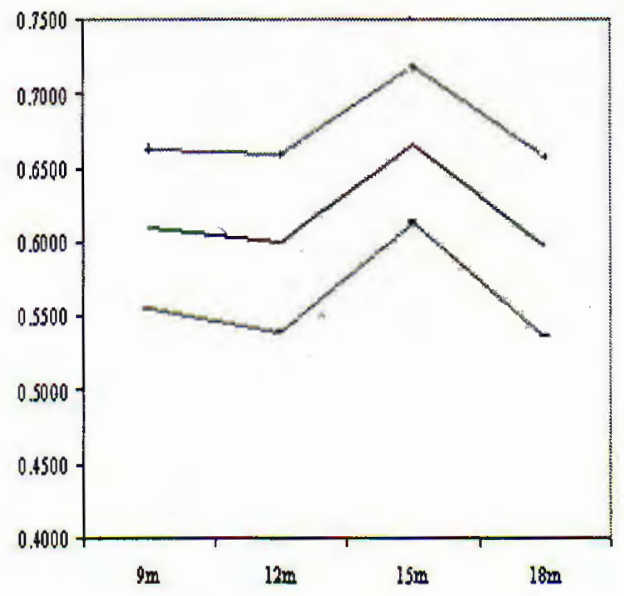

Gráfica 6. Análisis de sensibilidad de $\lambda_{11}$.

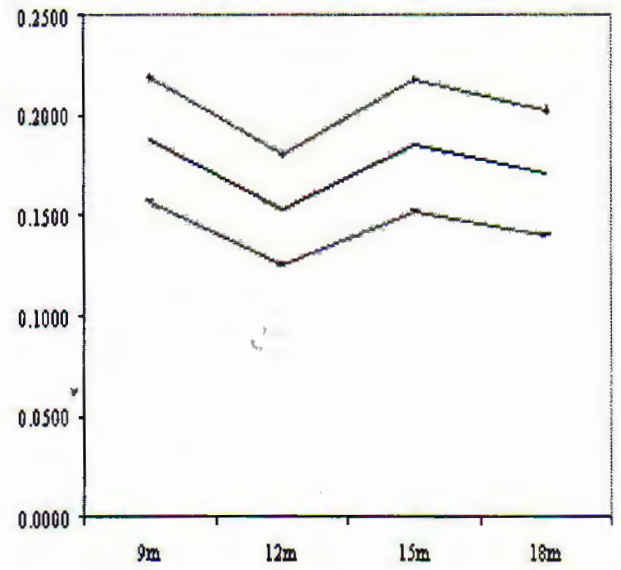

Asimismo, se observa que la correlación positiva se mantiene ante las distintas medidas de expectativas utilizadas para dividir en altos y bajos a los rendimientos, puesto que para cualquiera de esas medidas es mayor la probabilidad de obtener rendimientos bajos dados rendimientos bajos en los dos periodos anteriores que la de obtener altos. Las pruebas de hipótesis reportadas 
anteriormente para el promedio móvil a un año se mantienen en pie, rechazándose las hipótesis nulas de igualdad entre todas las probabilidades de transición y entre $\lambda_{00}$ y $\lambda_{11}$ para niveles de significancia marginal menores al $1 \%$. Con esto se puede concluir que los resultados de la prueba son robustos para la medida de expectativa utilizada.

\section{Probabilidades Estacionarias del Proceso}

De particular interés en el estudio de las cadenas de Markov es el cálculo de las probabilidades estacionarias del proceso. En el caso concreto de este trabajo, su importancia es crucial, ya que como se mencionó antes, las prueba sobre las cadenas de Markov no requieren que la serie de los rendimientos originales sea normalmente distribuida y ni siquiera estacionaria, siempre y cuando la cadena de Markov asociada sea estacionaria. Neftci (1984) désarrolla la expansión asintótica de la probabilidad del estado inicial, la que arroja como resultado las probabilidades estacionarias. El vector de probabilidades iniciales $\left[\pi_{00}, \pi_{01}, \pi_{10}, \pi_{11}\right]^{\prime}$ se relaciona con cuatro posibles eventos: $\left\{I_{2}=0, I_{1}=0\right\}$, $\left\{I_{2}=0, I_{1}=1\right\},\left\{I_{2}=1, I_{1}=0\right\}$ y $\left\{I_{2}=1, I_{1}=1\right\}$. Además, dado que las secuencias $\{1,1,1\}$ y $\{1,1,0\}$, así como $\{0,0,0\}$ y $\{0,0,1\}$, son eventos disjuntos, se tiene que:

$$
\begin{aligned}
\operatorname{Pr}\left\{I_{2}=1, I_{1}=1\right\}= & \operatorname{Pr}\left\{I_{2}=1 \mid I_{1}=1, I_{0}=1\right\} \operatorname{Pr}\left\{I_{1}=1, I_{0}=1\right\} \\
& +\operatorname{Pr}\left\{I_{2}=1 \mid I_{1}=1, I_{0}=0\right\} \operatorname{Pr}\left\{I_{1}=1, I_{0}=0\right\}
\end{aligned}
$$

$\mathrm{y}$

$$
\begin{aligned}
\operatorname{Pr}\left\{I_{2}=0, I_{1}=0\right\}= & \operatorname{Pr}\left\{I_{2}=0 \mid I_{1}=0, I_{0}=0\right\} \operatorname{Pr}\left\{I_{1}=0, I_{0}=0\right\} \\
& +\operatorname{Pr}\left\{I_{2}=0 \mid I_{1}=0, I_{0}=1\right\} \operatorname{Pr}\left\{I_{1}=0, I_{0}=1\right\} .
\end{aligned}
$$

Las restantes probabilidades se encuentran de manera similar obteniéndose el siguiente sistema de ecuaciones:

$$
\left(\begin{array}{l}
\pi_{11}(t) \\
\pi_{00}(t) \\
\pi_{10}(t) \\
\pi_{01}(t)
\end{array}\right)=\left(\begin{array}{cccc}
\lambda_{11} & 0 & \lambda_{10} & 0 \\
0 & \lambda_{00} & 0 & \lambda_{01} \\
0 & 1-\lambda_{00} & 0 & 1-\lambda_{01} \\
1-\lambda_{11} & 0 & 1-\lambda_{10} & 0
\end{array}\right)\left(\begin{array}{l}
\pi_{11}(t-1) \\
\pi_{00}(t-1) \\
\pi_{10}(t-1) \\
\pi_{01}(t-1)
\end{array}\right)
$$

Merece la pena detenerse un momento a observar la matriz de coeficientes del anterior sistema, en el cual se presenta una cadena de Markov de segundo orden bajo la forma de una cadena de primer orden, en la que tenemos una matriz de transición con cuatro estados. Antes, las probabilidades de transición estimadas eran para una cadena de segundo orden con dos estados. No obstante, con los presentes cuatro estados de esta cadena de primer orden es posible obtener información acerca de la cadena original de segundo orden. Este sistema se puede escribir como $\Pi(t)=A \Pi(t-1)$, que después de algunas sustituciones sucesivas da como resultado $\Pi(t)=A^{n} \Pi(T-n)$. El vector de probabilidades estacionarias se define como:

$$
\lim _{n \rightarrow \infty} \Pi(t)=\Pi^{*}
$$


La solución de este sistema de ecuaciones no es más que el vector de probabilidades estacionarias:

$$
\Pi^{*}=A \Pi^{*}, \quad 1^{\prime} \Pi^{*}=1
$$

donde $1=[1,1,1,1]^{\prime}$. Haciendo uso del hecho de que una de las ecuaciones del sistema es redundante, eliminamos la ecuación correspondiente a $\pi_{10}$ y obtenemos el siguiente sistema:

$$
\left(\begin{array}{cccc}
0 & 0 & \lambda_{10} & -\left(1-\lambda_{11}\right) \\
-\left(1-\lambda_{00}\right) & \lambda_{01} & 0 & 0 \\
0 & -1 & 1-\lambda_{00} & 1-\lambda_{11} \\
1 & 1 & 1 & 1
\end{array}\right)=\left(\begin{array}{l}
\pi_{00} \\
\pi_{01} \\
\pi_{10} \\
\pi_{11}
\end{array}\right)\left(\begin{array}{l}
0 \\
0 \\
0 \\
1
\end{array}\right)
$$

Después de resolver el sistema anterior, se calculan las probabilidades estacionarias en función de las probabilidades de transición de la siguiente forma:

$$
\begin{aligned}
\pi_{00} & =\frac{\left(1-\lambda_{11}\right) \lambda_{01}}{\Delta}, \\
\pi_{11} & =\frac{\left(1-\lambda_{00}\right) \lambda_{10}}{\Delta}, \\
\pi_{01}=\pi_{10} & =\frac{\left(1-\lambda_{11}\right)\left(1-\lambda_{00}\right)}{\Delta}
\end{aligned}
$$

donde

$$
\Delta=2\left(1-\lambda_{00}\right)\left(1-\lambda_{11}\right)+\lambda_{01}\left(1-\lambda_{11}\right)+\lambda_{10}\left(1-\lambda_{00}\right) .
$$

Al sustituir los valores estimados en la sección 3 en las probabilidades de transición, se obtienen las probabilidades estacionarias del proceso asignado a los rendimientos de la cartera estudiada en este trabajo:

$$
\pi_{00}=0.2624, \quad \pi_{11}=0.1975, \quad \pi_{01}=\pi_{10}=0.2699 .
$$

Las probabilidades estacionarias nos dan información acerca del estado inicial, y en este caso se repite el mismo patrón observado en las probabilidades de transición. Las probabilidades estacionarias se pueden ver como incondicionales, lo que se refleja en el hecho de que se agrupan en una matriz de $4 \times 1$, y no en una matriz de $4 \times 4$. Esta matriz nos da la probabilidad de permanecer en el estado $i j$ en todo tiempo, en particular, en el estado inicial y en el estado estacionario. Las probabilidades estacionarias refuerzan las conclusiones obtenidas en los anteriores apartados, puesto que nos dicen que en el estado estacionario (o alternativamente, en el estado inicial), es más probable estar en una secuencia de rendimientos bajos. Esto es, se encuentra evidencia de correlación positiva en las probabilidades estacionarias.

Como se mencionó antes, esta prưeba no requiere que la serie original de rendimientos sea estacionaria siempre y cuando la cadena de Markov asociada sea estacionaria. Esta información, la podemos obtener del análisis de los valores propios de la matriz de transición. La cadena es ergódica si los "unos" de los valores propios de la matriz son igual a la unidad y los restantes son menores 
a uno en valor absoluto (o en módulo, de ser valores complejos). Si la cadena es ergódica, el proceso es estacionario en covarianza.

La matriz de transición en este caso es de rango 4, por lo que esperamos encontrar 4 valores propios. Utilizamos el ordenamiento de columnas para encontrar las probabilidades estacionarias, bajo la condición de que los valores propios son invariantes ante permutaciones de las columnas de la matriz. El Cuadro 8 presenta los valores propios y sus valores absolutos.

Cuadro 8. Valores propios de la matriz de transición.

\begin{tabular}{|c|c|c|}
\hline Valor propio & Multiplicidad & Valor absoluto del módulo \\
\hline 1 & 1 & 1 \\
\hline 0.3294 & 1 & 0.3294 \\
\hline$-0.2886+0.4628 i$ & 1 & 0.5454 \\
\hline$-0.2886-0.4628 i$ & 1 & 0.5454 \\
\hline
\end{tabular}

La matriz tiene 4 valores propios distintos, dos reales y un conjugado complejo. En efecto, uno de los valores propios es uno y los restantes son menores a la unidad, ya sea en valor absoluto o en módulo, por lo que la cadena es ergódica y por tanto el proceso es estacionario en covarianza. De esta manera, concluimos que la prueba se encuentra bien especificada.

\section{Pronósticos}

Dado que la cadena de Markov utilizada para hacer la prueba es ergódica, es posible hacer pronósticos con ella. Con el ordenamiento de columnas usado para calcular las probabilidades ergódicas, se pueden indizar las columnas de la matriz de transición de acuerdo con los correspondientes estados. De esta manera, la primera columna se puede denotar como $(1,1)$, la segunda como $(0,0)$ y así sucesivamente. Consideremos ahora a $\epsilon_{t}$ como un vector aleatorio correspondiente a la columna $(i, j)$ de una matriz identidad de dimensión 4. Usando el ordenamiento de columnas descrito anteriormente, será posible lograr que la primera columna, correspondiente al estado $(1,1)$ sea un vector con un uno en la primera posición y ceros en las restantes.

Consideremos también, una realización de la cad"ena de primer orden, $S_{t}$, de tal forma que $S_{t}=i j, i, j=0,1$, el elemento correspondiente según el ordenamiento de columnas de la matriz identidad, es una variable aleatoria que toma el valor de la unidad con probabilidad $\lambda_{i j}$ ó $1-\lambda_{i j}$, dependiendo de su posición en la matriz de transición, y cero en otro caso. Esta variable aleatoria es un experimento de Bernoulli y, como tal, tiene un valor esperado igual a su probabilidad de tomar uno como valor. Bajo estas condiciones, la esperanza condicional de $\epsilon_{t+1}$ dado $S_{t}=i j$ viene dada por la correspondiente columna de la matriz de transición del proceso de Markov de primer orden, denotada como $L$. Dado que la realización es igual a la correspondiente columna de la matriz identidad de dimensión 4, se tiene que:

$$
\mathrm{E}\left[\epsilon_{t+1} \mid \epsilon_{t}, \epsilon_{t-1}, \ldots\right]=\mathrm{E}\left[\epsilon_{t+1} \mid \epsilon_{t}\right]=L \epsilon_{t}
$$


donde la primera igualdad se desprende de la propiedad Markoviana. De esta manera, es posible expresar la cadena de Markov de la siguiente manera:

$$
\epsilon_{t+1}=L \epsilon_{t}+\nu_{t+1}
$$

con

$$
\nu_{t+1}=\epsilon_{t+1}-\mathrm{E}\left[\epsilon_{t+1} \mid \epsilon_{t}, \epsilon_{t-1}, \ldots\right]
$$

donde la media de $\nu_{t}$ es un vector de ceros de dimensión 4 . El valor esperado de $\epsilon_{t+m}$ se puede obtener mediante sustituciones sucesivas, obteniendo lo siguiente:

$$
\epsilon_{t+m}=\nu_{t+m}+L \nu_{t+m-1}+L^{2} \nu_{t+m-2}+L^{3} \nu_{t+m-3}+\cdots+L^{m} \epsilon_{t} .
$$

Dado que la media de $\nu_{t}$ es cero, el valor esperado de $\epsilon_{\hat{t}+m}$ condicionado a la información en $t$ es igual a:

$$
\mathrm{E}\left[\epsilon_{t+m} \mid \epsilon_{t}, \epsilon_{t-1}\right]=L^{\dot{m}} \epsilon_{t} .
$$

De esta manera, se pueden pronosticar las probabilidades de transición de la cadena en $t+m$ elevando a la potencia $m$ la matriz de transición y multiplicandola por el correspondiente vector de la base canónica de $R^{4}$,(e), usando el ordenamiento de columnas de la matriz de la cadena de Markov de primer orden. Así, por ejemplo, el pronóstico $m$ periodos adelante dado que en $t$ la cadena se encontraba en el estado $S_{t}=11$ es igual a:

$$
\left(\begin{array}{l}
\operatorname{Pr}\left\{S_{t+m}=11 \mid S_{t}=11\right\} \\
\operatorname{Pr}\left\{S_{t+m}=00 \mid S_{t}=11\right\} \\
\operatorname{Pr}\left\{S_{t+m}=10 \mid S_{t}=11\right\} \\
\operatorname{Pr}\left\{S_{t+m}=01 \mid S_{t}=11\right\}
\end{array}\right)=L^{m} \mathrm{e}_{11} .
$$

Se demostró en la sección anterior que la cadena de Markov asociada a la serie de rendimientos es ergódica, por lo que se sabe que pasado un cierto lapso de tiempo, las probbabilidades pronosticadas tenderán a converger a sus probabilidades estacionarias. Las Gráficas 7 y 8 nos muestran los pronósticos para un horizonte de 30 días, dado que en $t$ la cadena se encontraba en el estado $S_{t}=11$ y $S_{t}=00$. Las gráficas están rotuladas como $P_{i j}$ de acuerdo con el estado alcanzado en $t+m$. 
Gráfica 7. Probabilidades pronosticadas para $t+m$ dado que $I_{t}=11$.

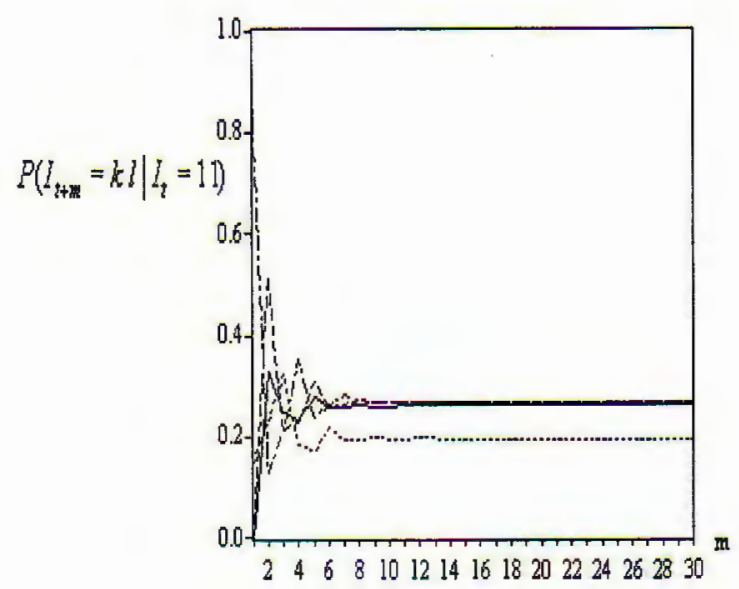

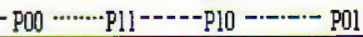

Gráfica 8. Probabilidades pronosticadas para $t+m$ dado que $I_{t}=00$.

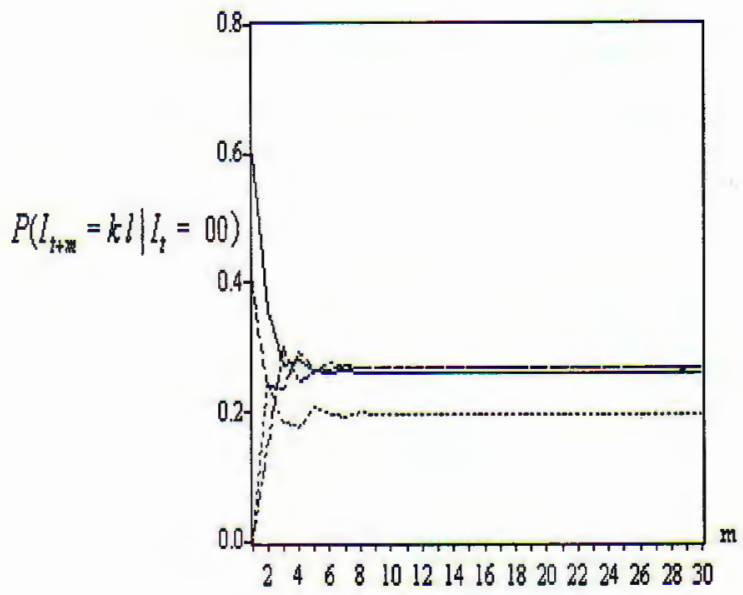

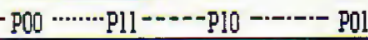

Se aprecia la rapidez con la que los pronósticos tienden a su media incondicional, esto es, a la probabilidad estacionaria del estado correspondiente. 
De acuerdo con la gráfica 8, la convergencia se alcanza alrededor de $m=26$, aunque muchos periodos atrás las fluctuaciones alrededor de la media incondicional son marginales. Este hecho indica que el horizonte de pronóstico de este modelo es muy corto, es decir, puede proporcionar predicciones inmediatas antes de asentarse en sus valores estacionarios. Esto no implica que la variable aleatoria se estacione, efectivamente, en esos valores, simplemente nos dice que después de cierto momento los pronósticos del modelo serán constantes. Entonces, teniendo en cuenta que los datos utilizados en este trabajo corresponden a días hábiles, el horizonte de pronóstico del modelo es aproximadamente de dos a tres semanas, alcanzándose hasta el mes la media incondicional.

\section{Conclusiones}

En el presente trabajo se probó la hipótesis de caminata aleatoria para los rendimientos nominales de una cartera de activos de alta bursatilidad con pesos iguales, expandiendo la metodología propuesta por McQueen y Thorley (1991). De esta manera, se asoció una cadena de Markov de segundo orden a la serie de rendimientos diarios de la cartera, y se encontró que la estructura de las probabilidades de transición de la cadena revela la existencia de una fuerte autocorrelación positiva. La significancia estadística de este resultado se probó mediante diversas comparaciones de los parámetros relevantes, encontrandose en todos, que la hipótesis nula de caminata aleatoria era enfáticamente rechazada. Los resultados de estas pruebas son robustos ante distintas medidas de expectativas, como lo señala el análisis de sensibilidad realizado, con lo cual se concluye que existen componentes predecibles en la serie de los rendimientos de la cartera aquí estudiada.

Asimismo, se calcularon las probabilidades estacionarias de la cadena, encontrándose evidencia de que la correlación positiva en las probabilidades de transición es un patrón que se repite en el estado estacionario. Por otro lado, se demostró que la cadena asociada a la serie de rendimientos es ergódica y por tanto estacionaria en covarianza, por lo que la prueba se encuentra bien especificada. Por último, se aprovechó la estimación usada en la prueba, y el hecho de que la cadena es ergódica, para hacer pronósticos sobre los estados futuros dada la información contenida en la matriz de transición estimada, y se observó que es posible obtener pronósticos con un horizonte de dos a tres semanas.

Se pueden señalar algunas limitaciones y extensiones a los resultados reportados antes. El uso de rendimientos diarios se prefirió en aras de la simplicidad computacional y la convergencia asintótica de los resultados. Un analisis similar usando rendimientos mensuales, por ejemplo, requeriría del estudio de las propiedades de muestra pequeña de los estimadores y las pruebas estadísticas utilizadas. McQueen y Thorley estiman la distribución empírica de sus estadísticos de prueba mediante simulación dado que el número de observaciones que utilizan es muy pequeño. Por otro lado, las probabilidades de transición obtenidas en este trabajo obvian las probabilidades del estado inicial puesto que se trabaja con una gran número de observaciones, pero la estimación para rendimientos mensuales requeriría de su tratamiento explícito. Asimismo, un 
análisis extensivo del mercado accionario mexicano implicaría la inclusión de activos menos bursátiles que los usados aquí, por lo que la correlación espuria debida al comercio infrecuente tendría que ser modelada explícitamente. Por último, los estudios sobre el tema señalan que el análisis de portafolios ponderados por tamaño puede inducir diferencias no triviales en los resultados, que no son captados por nuestro portafolio de pesos iguales. Todos estos refinamientos quedan como posibles extensiones al presente trabajo. De cualquier manera, el resultado principal que se obtiene es que se encontró evidencia de componentes pronosticables en los rendimientos de acciones de alta bursatilidad.

\section{Bibliografía}

Campbell, J. Y., A. W. Lo, and A. C. MacKinlay (1997). The Econometrics of Financial Markets. Princeton University Press, New Jersey.

Fama, E., and K. R. French (1988). Permanent and Temporary Components of Stock Prices. Journal of Political Economy, 96, pp. 246-273.

Falk, B. (1986). Further Evidence on the Asymetric Behavior of Economic Time Series over the Bussiness Cycle. Journal of Political Economy, 94, pp. 1906-1109.

Freund, J. E., and R. E. Walpole (1990). Estadística Matemática con Aplicaciones ( $4^{a}$ ed.). Prentice Hall, México.

Greene, W. H. (1999). Análisis Econométrico ( $3^{a}$ ed.). Prentice Hall, Madrid.

Grossman, S. I. (1996). Algebra Lineal ( $5^{a}$ ed.). McGraw Hill, Santa fe de Bogotá.

Hamilton, J.D. (1994). Time Series Analysis. Princeton University Press, New Jersey.

Kroll, Y., H. Levy, and A. Rapoport (1988). Experimental Tests of the mean-variance Model of Portfolio Selection. Organizational Behavior and Human Decision Process, 42, pp. 338-410. (citado por McQueen y Thorley (1991), página 242.)

Lo, A. W. (1997). Fat Tails, Long Memory and Stock Market since the 1960's. Economic Notes by Banca Monte de Paschi, 26(12), pp. 213-246.

Lo, A. W., and A. C. MacKinlay (1988). Stock Market Prices do not Follow Random Walks: Evidence from a Simple Specification Test. Review of Financial Studies, 1, pp. 41-66.

Lorenzo-Valdés, A. (2002). Pruebas de No-Linealidad de los Rendimientos del Mercado Mexicano Accionario: Coeficientes de Lyapunov. Por aparecer en Estudios Económicos.

McQueen, G. Y., and S. Thorley (1991). Are Stock Returns Predictable? A test Using Markov Chains. Journal of Finance, 56, pp. 239-263.

Neftci, S. N. (1984). Are Economic Time Series Asymmetric over the Bussiness Cycle?. Journal of Political Economy, 92, pp. 307-328.

Ramírez, J. C. (2001). Los Problemas más Comunes en el Pronóstico de Rendimientos de Activos Financieros. Documento de Trabajo No. 204. Centro de Investigación y Docencia Económicas, México.

Venegas-Martínez, F. (2001). Opciones, Cobertura y Procesos de Difusión con Saltos: Una Aplicación a los Títulos de G-CARSO. Estudios Económicos, 16(2), pp. 203-226. 